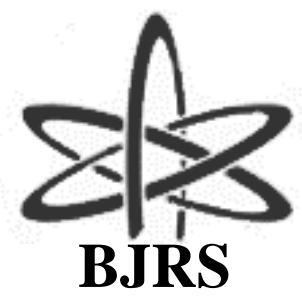

\author{
BRAZILIAN JOURNAL \\ $\mathrm{OF}$ \\ RADIATION SCIENCES \\ 09-02B (2021) 01-12
}

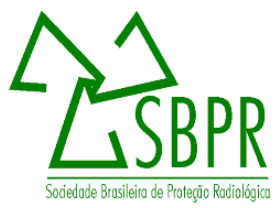

\title{
A Method for the evaluation of knowledge
}

\section{management systems}

\author{
Grecco $^{a}$ C.H.S., Augusto ${ }^{a}$ S.C., Souza ${ }^{a}$ J.T., Carvalho ${ }^{a}$, P. V. R, d'Avila ${ }^{a}$, A. L. \\ ${ }^{a}$ Departamento de Engenharia Nuclear/Serviço de Engenharia de Sistemas Complexos/Instituto de Engenharia \\ Nuclear(IEN/CNEN), 21941-906, Rio de Janeiro-RJ, Brasil grecco@ien.gov.br
}

\begin{abstract}
The explicit and tacit knowledge of workers constitute as valuable resources, as they enable organizations to perform their functions successfully. Such knowledge is derived from the qualification and experience of workers. On the other side, there are factors that favor the loss of this knowledge in organizations, as for example, the natural aging of workers and consequently the retirement and staff turnover. Because of these reasons, it becomes important for any organization to seek the preservation of its explicit and tacit knowledge. The Knowledge Management seeks to assist in the tasks of identification, organization, preservation and transmission of such knowledge. At the Nuclear Engineering Institute, three systems were developed and implemented to help in the Knowledge Management area: CarpeDIEN, a repository of research data based on the DSpace software; the "Nuclear Energy in Magazines", a peer-reviewed academic journals management system based on the Open Journal Systems software; and WikiIEN, a wiki based on the MediaWiki software. Each of these systems presents a different proposal and workflow. The objective of this paper is to present the characteristics of each system, a method to compare them based on criteria presented in the literature and to point their weaknesses. The results showed that the method is a proactive tool to provide a basis for choosing the most appropriate system for a given need in an organization.
\end{abstract}

Keywords: knowledge management, evaluation methods, systems. 


\section{INTRODUCTION}

Knowledge management is the process of creating, storing, managing, using and sharing the knowledge and information of an organization [1]. It refers to a multidisciplinary approach (including the fields of business administration, information systems, management, library, and information sciences) to achieving organizational objectives by making the best use of knowledge [2][3][4]. It is an important tool for the preservation of the knowledge and information of an organization, and for its transference from older to newer staff. Many large companies, public institutions and non-profit organizations have resources dedicated to internal knowledge management (KM) efforts, often as a part of their business strategy, information technology, or human resource management departments [5].

Several information systems can be employed on an organization to assist on its efforts on KM. On the Nuclear Engineering Institute (IEN), a Brazilian federal research institute of the Nuclear Energy National Commission (CNEN), three systems are in use on this field: WikiIEN, Nuclear Energy in Magazines and CarpeDIEN. These systems are web applications, a kind of software that runs on a combination of web server (the processing and data storage side) and web browser (the user interface side) over a computer network, such as the internet or the IEN's intranet network [6]. Each of these web applications have different purposes and therefore function differently from each other.

Thus, the purpose of this article is to compare the functionality of each of these web applications using a single methodology, pointing the verified strengths and weaknesses of each one regarding their use on knowledge management. First, each web application will be briefly reviewed. Then, the methodology will be presented and applied to evaluate the functionality of the KM systems. Finally, a comparison of the results will be shown and commented.

\subsection{WikiIEN}

WikiIEN is a wiki-type web application based on the MediaWiki software. It enables the users to create and modify articles on a WYSIWYG ("What You See Is What You Get") editor with text, links to another articles on the same wiki or to web sites, mathematical formulae, images and 
videos. All users are required to log in to be able to create / edit articles, a relevant point because of the application's serious purpose in a research institute. The content is focused on nuclear knowledge and/or IEN-related. Its purpose is to enable the users to write new article and to improve the existing ones collaboratively, in order to preserve the knowledge of workers and collaborators from IEN. It uses the following MediaWiki extensions: Confirm User Accounts, User Merge and Delete, WYSIWYG editor, SimpleMathJax, and HTML5video.

Originally, MediaWiki didn't have a WYSIWYG editor, requiring contributors to create / edit articles in wikitext, a markup language somewhat comparable to HTML, and so not user-friendly for most users, who are used to WYSIWYG-like editors like Microsoft Word. The WikiIEN project was halted until MediaWiki 's own WYSIWYG editor was ready.

A wiki is a knowledge base website on which users collaboratively modify content and structure directly from the web browser. In a typical wiki, text is written using a simplified markup language and often edited with the help of a rich-text editor [7]. MediaWiki is a free, open-source wiki-type web application widely used, optimized to handle large-scale projects, with terabytes of content and hundreds of thousands of hits per second, more than 700 configuration options and more than 1800 extensions [8]. Wikipedia is the most famous website that uses MediaWiki. It was originally developed in 2002 by the Wikimedia Foundation to meet the needs of Wikipedia, which has been used by a hundred companies as an internal knowledge management system, including Intel, Novell, and the corporate university of Banco do Brasil [9].

MediaWiki has a skin feature, which enables the user to choose one of several profiles that modify the appearance and layout of the page, such as presence/absence, positioning, dimension and color of page elements. The skin used in the WikiIEN is the default skin of MediaWiki and Wikipedia.

\subsection{Nuclear Energy in Magazines (NEM)}

The Nuclear Energy in Magazines (NEM) is a web application for the management of peerreviewed, nuclear technology-related academic journals. It is based on the Open Journal Systems software. Currently, it features the "Nuclear Engineering Institute: Progress Report" academic journal, which publish articles about scientific research done at the IEN. As of 2019, it's at its 3rd 
edition, with the three editions covering works from the years 2011 to 2017. A 4th edition is planned for the year 2020, covering works from the years 2018-2019.

Open Journal Systems (OJS) is open-source software created by the Public Knowledge Project (PKP) for the management of peer-reviewed academic journals. It facilitates the publishing process by providing the technical infrastructure for both the online presentation of journal articles and for the entire editorial management workflow, including: article submission, multiple rounds of peerreview, and indexing. For OJS to operate as intended, its users must fulfill their roles properly, such as: the journal manager (system administration), the editor (direct new article submissions to reviewers, accept or reject article submission), the reviewer (evaluate the article submission, point needed corrections), the author (submit article, make needed corrections) and the reader (access articles accepted in all evaluation stages) [10]. It has a module that supports subscription journals.

The software has a 'plugin' architecture that allows new features to be integrated. OJS is also LOCKSS-compliant, helping to ensure permanent archiving for ongoing access to the content of the journal.

As of mid-2015, OJS was being used by at least 8,000 journals worldwide. A map showing the location of these journals is also available on PKP 's website. A survey in 2010 found that about half were in the developing world. OJS is being used in the Synergies project, creating a scholarly portal for Canadian social sciences and humanities research, and also for research portals in Brazil, Spain, Italy and Greece.

\subsection{CarpeDIEN}

CarpeDIEN is a web application based on the DSpace software. It serves as a repository to articles, data files (such as data tables) and metadata about scientific research developed by IEN workers and collaborators.

DSpace is an open source repository software package typically used for creating open access repositories for scholarly and/or published digital content. While DSpace shares some feature overlap with content management systems and document management systems, the DSpace repository software serves a specific need as a digital archives system, focused on the long-term storage, access and preservation of digital content [11]. 
The first public version of DSpace was released in November 2002, as a joint effort between developers from MIT and HP Labs. Currently the DSpace software and user community receives leadership and guidance from DuraSpace, a not-for-profit organization.

DSpace is constructed with Java web applications, many programs, and an associated metadata store. The web applications provide interfaces for administration, deposit, ingest, search, and access. The asset store is maintained on a file system or similar storage system. The metadata, including access and configuration information, is stored in a relational database and supports the use of PostgreSQL and Oracle database. DSpace holdings are made available primarily via a web interface. Some most important features of DSpace are as follows:

- Completely customizable to fit user needs;

- Manage and preserve all format of digital content (PDF, Word, JPEG, MPEG, TIFF files);

- Granular group based access control, allowing setting permissions down to the level of individual files;

- Optimized for Google Scholar indexing.

Notable DSpace repositories:

- The World Bank - Open Knowledge Repository;

- Apollo - University of Cambridge Repository;

- Digital Access to Scholarship at Harvard;

- DSpace@MIT;

- Spiral - Imperial College London Repository;

- WHO Institutional Repository for Information Sharing;

\section{MATERIALS AND METHODS}

In the last two decades, several software packages were developed specifically for use in the KM field. At the same time, software originally developed for other fields, such as process modeling and database management, were eventually branded as KM software. Added to a lack of 
adequate criteria, the commonly vague understandings of $\mathrm{KM}$ concepts make difficult the task of choosing the most appropriate software for a given need in an organization [12].

Several studies presented criteria and information to assist in the evaluation and choice of the most appropriate KM system for a given use [12][13][14][15]. Evaluation criteria usually focus on software functionalities grouped in categories, such as: usability, search features, user customization, content management, online collaboration and administration. The redundancy, complexities and abstract definitions found in each KM system brings difficulties in properly using a given set of criteria. Therefore, the characteristics and functionalities are considered in the criteria so that the most adequate KM system is chosen to serve the institutional needs. The evaluation of such criteria is subjective, and based on the evaluators' experience and technical knowledge while using such systems.

The methodology in use is the field research, in which phenomena are analyzed as they naturally occur [16]. The research carried out is of participant-observer type, that the researcher acts in relation to the effects of the phenomena studied [16]. The method of evaluation was created and used by the researchers and authors of this work. The evaluation of WikiIEN, Nuclear Energy in Magazines and CarpeDIEN systems was structured in two steps:

1. Selection criteria;

2. Evaluation of systems by experts

\subsection{Selection criteria}

The criteria used in this work consists of a list of 14 criteria, classified into 7 categories, based on literature [12][14][15]. The separation of criteria into categories allows perceiving the level of development of each system in relation to a broader set of criteria. The categories were drawn up to reflect functionality, demonstrating positive aspects (strengths) and negative aspects (weaknesses) of each system. The categories, criteria and descriptions are presented in Table 1.

WikiIEN, Nuclear Energy in Magazines and CarpeDIEN are evaluated by experts in KM systems using the criteria presented on table 1 . The objective of this evaluation is to verify whether the systems meet the criteria. In this step is performed a comparison between the systems. 
Table 1: Classified criteria in categories.

\begin{tabular}{lll}
\hline Categories & \multicolumn{1}{c}{ Criteria } & \multicolumn{1}{c}{ Descriptions } \\
\hline 1. Usability & 1.1 Ease of use & How easily system is operated by user. \\
\hline $\begin{array}{l}\text { 2. Categorization } \\
\text { and Taxonomy }\end{array}$ & 2.1 Metadata & $\begin{array}{l}\text { Data that provides information about } \\
\text { other data. }\end{array}$ \\
\cline { 2 - 3 } & 2.2 Categories & Troup content by affinity. \\
\hline 3. Search Features & 3.1 Search engine & Tool for searching data on system. \\
\cline { 2 - 3 } & 3.2 Scope of search results & Search includes data and metadata. \\
\cline { 2 - 3 } & 3.3 Presentation of search results & $\begin{array}{l}\text { Appropriate presentation of search } \\
\text { results. }\end{array}$ \\
& &
\end{tabular}

\section{User Customiza-}

tion 4.1 Layout Customization

Ability to change the system interface.

5. Content Mana- $\quad 5.1$ Content creation and visualization gement
Create and view data and metadata in-system.

5.2 Rules of publication and access

Change of user permissions or or restrictions.

5.3 Changes Management

Ability to revert content changes.

5.4 Integration

Ability to connect to other systems.

6. Online Collaboration 6.1 Online discussion tools Tools for users to discuss content.

7. Administration 7.1 Management of communities Create groups, add or remove or groups users to them.

7.2 Administrative tools Tools that enable administrative activities. 


\section{RESULTS AND DISCUSSION}

WikiIEN, Nuclear Energy in Magazines (NEM) e CarpeDIEN were evaluated by five experts (authors of this work). The results were obtained from a consensus of opinions among the experts. The results are presented on table 2 .

Table 2: Evaluation of WikiIEN, NEM and CarpeDIEN systems.

\begin{tabular}{|c|c|c|c|c|}
\hline \multirow{2}{*}{$\begin{array}{l}\text { Categories } \\
\text { 1. Usability }\end{array}$} & \multirow{2}{*}{$\begin{array}{c}\text { Criteria } \\
1.1 \text { Ease of use }\end{array}$} & \multirow{2}{*}{$\begin{array}{c}\text { WikiIEN } \\
X\end{array}$} & \multirow[t]{2}{*}{ NEM } & \multirow[t]{2}{*}{ CarpeDIEN } \\
\hline & & & & \\
\hline \multirow{2}{*}{$\begin{array}{l}\text { 2. Categorization } \\
\text { and Taxonomy }\end{array}$} & 2.1 Metadata & $X$ & $X$ & $X$ \\
\hline & 2.2 Categories & $X$ & $X$ & $X$ \\
\hline \multirow[t]{3}{*}{ 3. Search Features } & 3.1 Search engine & $X$ & $X$ & $X$ \\
\hline & 3.2 Scope of search results & $X$ & & \\
\hline & 3.3 Presentation of search results & $X$ & $X$ & $X$ \\
\hline \multicolumn{2}{|c|}{ 4. User Customization 4.1 Layout Customization } & $X$ & $X$ & $X$ \\
\hline \multirow{4}{*}{$\begin{array}{l}\text { 5. Content Mana- } \\
\text { gement }\end{array}$} & \multicolumn{4}{|l|}{ 5.1 Content creation and visualization $X$} \\
\hline & 5.2 Rules of publication and access & $X$ & $X$ & $X$ \\
\hline & 5.3 Changes Management & $X$ & $X$ & $X$ \\
\hline & 5.4 Integration & $X$ & $X$ & $X$ \\
\hline \multicolumn{2}{|c|}{ 6. Online Collaboration 6.1 Online discussion tools } & $X$ & & \\
\hline \multirow[t]{3}{*}{ 7. Administration } & 7.1 Management of communities & & & \\
\hline & or groups & $X$ & $X$ & $X$ \\
\hline & 7.2 Administrative tools & $X$ & $X$ & $X$ \\
\hline
\end{tabular}

This result showed that four criteria are not presented by all systems. The problems related to four criteria are commented below: 
- "Ease of use" criterion: WikilEN is based on the MediaWiki software, which is developed by Wikimedia Foundation for use on Wikipedia, which is aimed at the general public, so its user interface was designed to be more user-friendly from start, and has received large user feedback over a long time. On the other hand, Nuclear Energy in Magazines and CarpeDIEN are based on the OJS and DSpace software respectively, which are directed toward niche communities. And, in OJS' case, it uses an editorial management workflow, which is uncommon for the general public.

- "Scope of search results" criterion: Content in WikiIEN (e. g. text entered by the enduser) is directly stored into the system's relational database, so its search engine can search for keywords in articles and metadata. Content in Nuclear Energy in Magazines and CarpeDIEN is stored in files generated by external application software. These files cannot be searched for keywords by their search engine, which are restricted to metadata, resulting in a more restricted search scope.

- “Content creation and visualization" criterion: WikiIEN provides means to create and to visualize data and metadata, making it less dependent on external software. Nuclear Energy in Magazines and CarpeDIEN provide means to create and visualize metadata only, thus requiring external application software to create and to visualize data. Some web browser features can supplement this lack of functionality from the systems.

- "Online discussion tools" criterion: WikiIEN is focused at a community of users who collaborate more closely between themselves to aggregate knowledge. It needed better online discussion tools if compared to Nuclear Energy in Magazines and CarpeDIEN. It even enables users to discuss individual articles and comment each article modification. Nuclear Energy in Magazines and CarpeDIEN, which are aimed at aggregating documents d articles/works from authors or groups of authors, didn't need so detailed discussion tools. 


\section{CONCLUSION}

We proposed a method that uses criteria, classified into 7 categories, and the judgment of the experts in KM systems. These categories were drawn up to reflect the functionality of the systems. A pilot study in WikiIEN, Nuclear Energy in Magazines and CarpeDIEN shows that this method based on criteria offers interesting perspectives to compare these systems, to point weaknesses of each system and to choose the most appropriate system for a given need in IEN.

The results point out that WikiIEN is a solution more indicated for a bigger, general public because of its more user-friendly interface and workflow, auto-sufficient set of features not dependent on external software. Nuclear Energy in Magazines and CarpeDIEN are solutions more indicated for smaller, niche public because of a less user-friendly interface, workflow directed at individual or small group of users who uses specialized software.

Using this method we identify problems related to four criteria: "Ease of use", "Scope of search results", "Content creation and visualization" and "Online discussion tools". These specific problems pointed weaknesses in Nuclear Energy in Magazines and CarpeDIEN.

As suggestions for future research, we highlight: (1) the development of a computational system in order to automate the use of the method to evaluate a KM system online; (2) the periodic application of the method to estimate how changes made on these systems improved their performance. 


\section{REFERENCES}

[1] GIRARD, J.P.; GIRARD, J.L. Defining knowledge management: Toward an applied compendium. Online Journal of Applied Knowledge Management. 3 (1): 14, 2015.

[2] UNIVERSITY OF NORTH CAROLINA AT CHAPEL HILL. Introduction to Knowledge Management. Available in: www.unc.edu. Last accessed: 11 April 2019.

[3] NONAKA, I. The knowledge creating company. Harvard Business Review, pp. 96-104, 1991.

[4] NONAKA, I.; GEORGE, V. K. Tacit Knowledge and Knowledge Conversion: Controversy and Advancement in Organizational Knowledge Creation Theory. Organization Science, 20, pp. 635-652, 2009.

[5] ADDICOT, R.; MCGIVERN, G.; FERLIE, E. Networks, Organizational Learning and Knowledge Management: NHS Cancer Networks. Public Money \& Management, 26(2), pp. 87-94, 2006.

[6] WIKIPEDIA. Web application. Available at: <https://en.wikipedia.org/wiki/Web_application>. Last accessed: 30 March 2019.

[7] WIKIPEDIA. Wiki. Available at: <https://en.wikipedia.org/wiki/Wiki>. Last accessed: 30 March 2019.

[8] MEDIAWIKI. Mediawiki home page. Available at: < https://www.mediawiki.org/>. Last accessed: 11 April 2019.

[9] WIKIPEDIA. Mediawiki. Available at: < https://en.wikipedia.org/wiki/MediaWiki>. Last accessed: 11 April 2019.

[10] WIKIPEDIA. Open Journal Systems. Available at: < https://en.wikipedia.org/wiki/Open_Journal_Systems>. Last accessed: 10 April 2019.

[11] WIKIPEDIA. Dspace. Available at: <https://en.wikipedia.org/wiki/DSpace>. Last accessed: 6 April 2019. 
[12] TERRA, J. C.; GORDON, C. Portais Corporativos: a Revolução da Gestão do Conhecimento. São Paulo: Negócio Editora, 2002.

[13] CARVAlHO, R. B. Aplicações de Softwares de Gestão do Conhecimento: tipologia e usos. Dissertação de mestrado. Programa de Ciência da Informação. Universidade Federal de Minas Gerais, 2001.

[14] SAFA, N. S; VON SOLMS, R. An information security knowledge sharing model in organizations. Computers in Human Behavior, 57, pp. 442-451, 2016.

[15] ROBERTSON, J. How to evaluate a content management systems. Available at: <https://www.steptwo.com.au/papers/kmc_evaluate/>. Last accessed: 13 April 2019.

[16] DANE, F. C. Research Methods. California: Brooks / Cole Publishing Company, 1991. 